\title{
Subarachnoid Serotonergic and Noradrenergic Antagonists increase the Pain Response in Rats
}

\author{
Oscar César Pires, TSA ${ }^{1}$, Hazem Adel Ashmawi, TSA 2 , Elton Constantino ${ }^{3}$, Naira Correa Cusma Pelogia ${ }^{4}$, \\ Irimar de Paula Posso, TSA 5
}

Summary: Pires OC, Ashmawi HA, Constantino E, Pelogia NCC, Posso IP - Subarachnoid Serotonergic and Noradrenergic Antagonists Increase the Pain Response in Rats.

Background and objectives: There is evidence that the passage of nociceptive information through the posterior horn of the spinal cord (PHSC) on its way to rostral levels of the central nervous system undergoes profound excitatory and inhibitory influences. The objective of the present study was to compare the effects of the subarachnoid administration of methysergide, phentolamine, and phentolamine associated with methysergide on phases I, intermediate, and II of the modified phormaline test in rats.

Methods: Twenty-eight male Wistar rats distributed randomly in four groups $(n=7)$ to received subarachnoid saline solution (GC), phentolamine (GF), methysergide (GM), or phentolamine associated with methysergide (GFM). Pain was induced by the administration of phormaline in the dorsal region of the right hind paw. The test was divided in three phases: phase I, intermediate, and phase II. Statistical analysis of the results was performed using the software SPSS (Statistical Package for Social Sciences), adopting a level of significance of 5\%.

Results: In the intermediate phase the number of paw elevations was significantly higher in GF, GM, and GFM groups when compared to the GC group.

Conclusions: The results suggest the existence of a noradrenergic and serotonergic effect in the inhibitory descending system of acute pain, with the possibility of using serotonergic and $\alpha 1$-adrenergic antagonists to control acute pain.

Keywords: Pain measurement; Subarachnoid Space; Phentolamine; Methysergide; Rats.

[Rev Bras Anestesiol 2011;61(2): 202-210] CElsevier Editora Ltda.

\section{INTRODUCTION}

Nociception is a bidirectional process with ascending and descending pathways in the nervous system. Pain signals processed in the periphery go through the posterior horn of the spinal cord (PHSC) on their way to cerebral centers and, in response, projections leave the brain to the PHSC making several connections ${ }^{1}$. The proposed descending pain inhibitory system consists of areas of the central nervous system (CNS) interconnected to fibers that leave the cortical and diencephalic systems to the periaqueductal (PAG) and

Received from Instituto Básico de Biociências - Universidade de Taubaté (UNITAU), Taubaté, São Paulo, Brazil.

1. Responsible for the CET/SBA of Hospital Municipal de São José dos Campos, SP; PhD in Anesthesiology; Master's Degree in Pharmacology and Professor of Anesthesiology and Pharmacology - UNITAU

2. Professor of the Departamento de Medicina do Centro de Ciências Biológicas e da Saúde of Universidade Federal de São Carlos; Collaborating Professor of the Departamento de Cirurgia of Faculdade de Medicina da Universidade de São Paulo (FM/USP) and Laboratório de Investigação Médica - LIM-08 of FMIUSP

3. Anesthesiologist; Professor of Anesthesiology of UNITAU

4. Professor of Physiology and Pharmacology of UNITAU; PhD in Pharmacology; Master's Degree in Pharmacology

5. Professor of Disciplina de Anestesiologia of FMIUSP; Professor of Pharmacology of UNITAU

Submitted on July 4, 2010

Approved on December 13, 2010.

Correspondence to:

Dr. Oscar César Pires

Avenida Itália, 1.551 - Rua 13, $n^{\circ} 821$

12030-212 - Taubaté, SP, Brazil

E-mail: ocpires@uol.com.br periventricular gray matter, which are rich in enkephalins and opioid receptors and, from there on, they go to areas of the rostroventral medulla, especially the nucleus raphe magnus (NRM) and adjacent nuclei that send serotonergic and noradrenergic fibers, via the dorsolateral funiculus to the dorsal horn of the spinal cord and medulla where they end, mainly in laminae I, II, and V inhibiting nociceptive neurons, interneurons, and ascending tracts that project rostrally including the spinothalamic, spinoreticular, and spinomesencephalic tracts ${ }^{1-3}$. Therefore, it is expected that the increase in noradrenalin, serotonin, and opioid neurotransmission could be effective in pain control.

Phentolamine is an imidazole derivative that has competitive antagonist activity with affinity similar for both subtypes of $\alpha 1$ and $\alpha 2$ adrenergic receptors, affecting the pain phenomenon ${ }^{4}$.

Methysergide, a butanolamine of 1-methyl-d-lisergic acid, is a non-specific serotonin receptor antagonist used in several animal studies on serotonergic pain modulation ${ }^{5}$.

The objective of the present study was to evaluate the effects of the subarachnoid administration of the adrenergic antagonist, phentolamine, serotonergic antagonist, methysergide, and the association of both antagonists on modulation of pain induced by the modified phormaline test.

\section{METHODS}

Twenty-eight Wistar rats weighing 220 to 300 grams, furnished by the Experimentation Biotherium of the university 
were used. Three animals were kept per compartment, where they remained for at least 15 days before the beginning of the experiment for adequate adaptation. They were treated with balanced commercial feed and water ad libitum, 12-hour daynight cycle, and temperature of $22^{\circ} \mathrm{C} \pm 3^{\circ} \mathrm{C}\left(19^{\circ} \mathrm{C}\right.$ to $\left.25^{\circ} \mathrm{C}\right)$.

To administer the drugs in the subarachnoid space, animals were anesthetized with halothane at a 3.0\% concentration in inspired oxygen fraction of 1.0, administered by a calibrated vaporizer, and maintained for enough time to the animal to lose postural reflexes and become unable to walk in the chamber. At that moment, the animal was removed from the chamber and placed with its head in a cone-shaped mask through which it received the same concentration of halothane and oxygen.

After tricotomy, a transversal incision was performed in the midline of the intervertebral space above the next to last lumbar vertebra. Through the incision, the subarachnoid space was punctured with a 22G Tuohy needle, which was identified by reflex movements of the tail or hind legs. After the identification of the subarachnoid space, a Teflon PE-10 catheter was introduced through the needle for the administration of drugs. Before suturing the skin, the catheter was fixed on the musculature to confirm its location inside the subarachnoid space at the end of the procedure, after the animal was killed.

Animals were divided in four groups of seven rats each. Animals in the control group (GC) received $10 \mu \mathrm{L}$ saline; in the phentolamine group (GF), animals received 82 nmoles of phentolamine diluted in $10 \mu \mathrm{L}$ of saline; in the methysergide group (GM), animals received 64 nmoles of methysergide in $10 \mu \mathrm{L}$ of saline; and in the phentolamine associated with methysergide group (GFM), they received 82 nmoles of phentolamine associated with 64 nmoles of methysergide in $10 \mu \mathrm{L}$ of saline. The volumes and doses were defined based on prior studies ${ }^{4,6}$. All drugs were obtained from the SigmaAldrich laboratory, Saint Louis, MO, USA.

Pain was induced by injecting $50 \mu \mathrm{L}$ of $2 \%$ phormaline solution in the dorsal region of the right hind paw 25 minutes after the administration of the drugs in the subarachnoid space.

Paw elevations not related to ambulation during a period of 60 minutes were recorded, and the number of partial elevations was recorded every 5 minutes.

The test was divided in three phases: phase I, intermediate, and phase II. In phase I, the number of elevations in the first five minutes was recorded; in the intermediate phase, the number of paw elevations from the $6^{\text {th }}$ to the $20^{\text {th }}$ minute was recorded; and in phase II, the number of paw elevations from the $21^{\text {st }}$ minute to the $60^{\text {th }}$ minute was recorded.

The software SPSS (Statistical Package for Social Sciences), version 13.0, was used for the statistical analysis of the results adopting a level of significance of $5 \%(0.05)$. To identify differences in each group, the Wilcoxon Signed Rank Test was used. To identify differences among the four groups, the Kruskal-Wallis Test was used and whenever a difference among the groups was observed the Mann-Whitney Test was used to identify which groups differed.

\section{RESULTS}

In all groups, a biphasic behavior with an intermediate phase significantly different than phases I and II (Figure 1) was observed. In phase I, significant differences were not observed in the number of paw elevations among the four groups (Figure 2).

In the intermediate phase, the number of paw elevations was significantly greater in GF, GM, and GFM groups when compared to the GC group; however, a difference was not observed when the GF, GM, and GFM groups were compared to each other (Figure 3), although the number of paw elevations was greater in the GFM group, in which the serotonergic and noradrenergic antagonists were associated, than in the GF and GM groups, in which they were used isolatedly. The GM group showed a smaller number of paw elevations than the GF and GFM groups; however, this difference was not statistically significant.

In phase II, significant differences were not observed when groups GF, GM, and GFM were compared to GC group; however, when the GM group was compared to GF and GFM, it showed a statistically significant reduction in the number of paw elevations (Figure 4).

\section{Mean paw elevations}

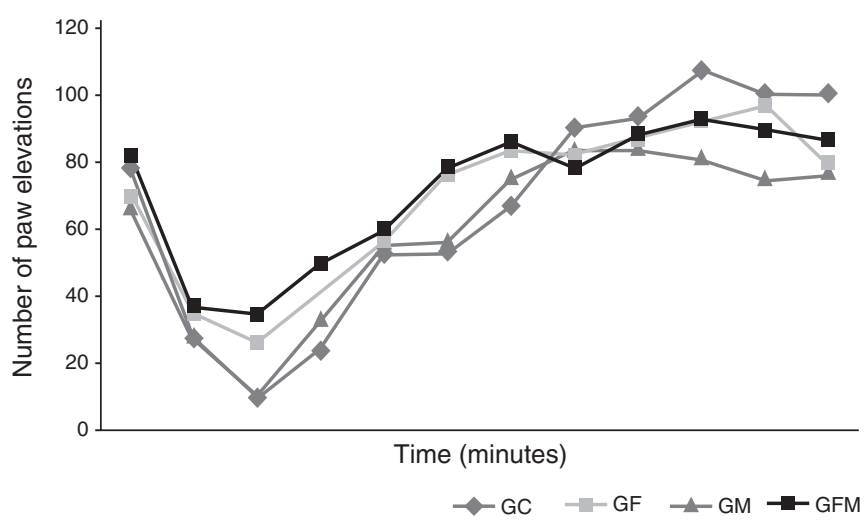

Figure 1. Mean Number of Paw Elevations During all the Phases of the Modified Phormaline Test in all Groups.

Phase I

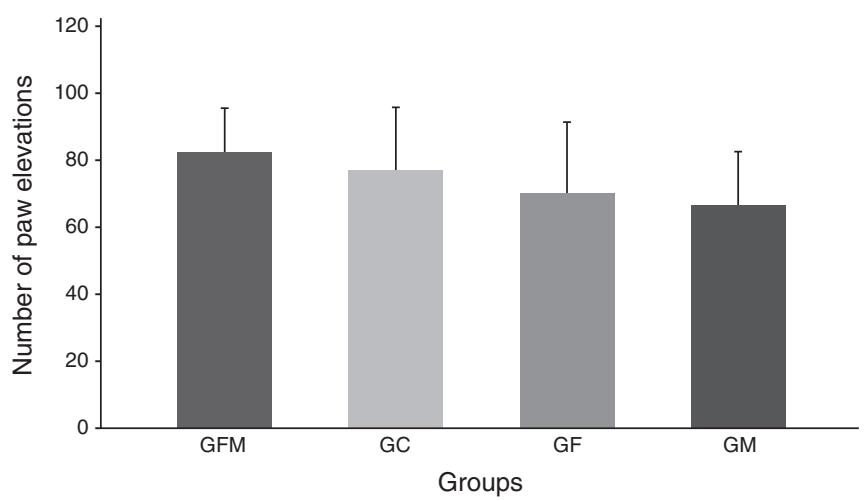

Figure 2. Means and Standard Deviation of the Number of Paw Elevations in all Groups during Phase I of the Modified Phormaline Test. No significant differences among groups. 


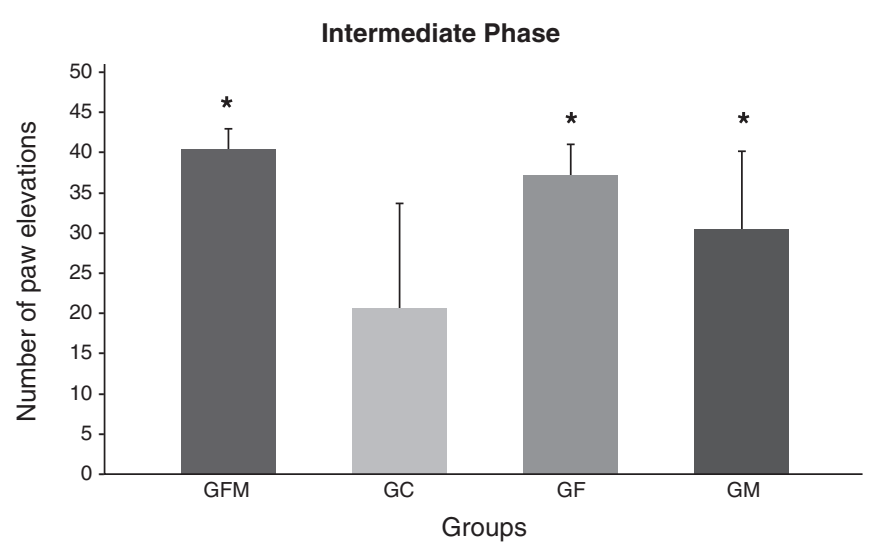

Figure 3. Means and Standard Deviation of the Number of Paw Elevations in all Groups during the Intermediate Phase of the Modified Phormaline Test.

* Statistically significant difference between groups GC $\times$ GFM ( $p=0.025)$; GC $x$ GF $(p=0.025)$; and GC x GM $(p=0.047)$.

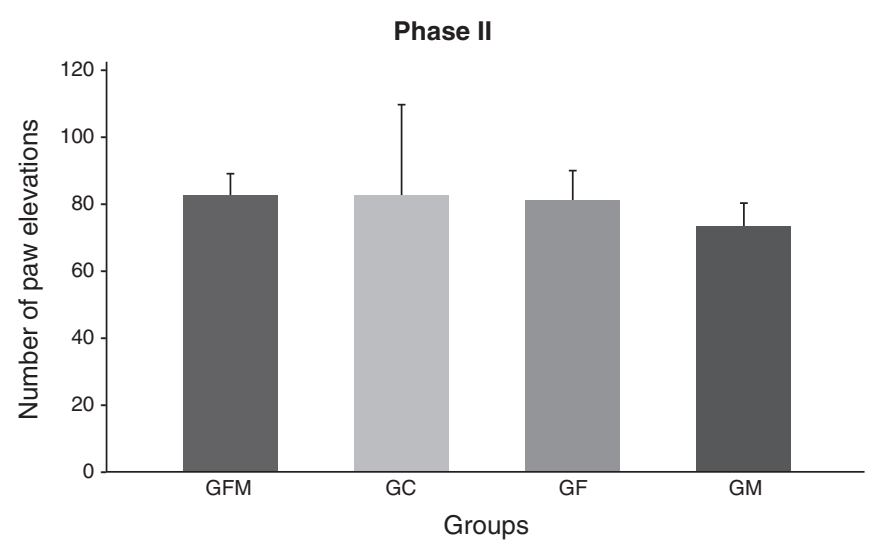

Figure 4. Means and Standard Deviation in all Groups during Phase II of the Modified Phormaline Test.

*Statistically significant difference between groups GM $\times$ GFM ( $p=0.013)$ and GM $\times$ GF $(p=0.035)$.

\section{DISCUSSION}

The phormaline test is an old experimental model of pain in animals that consists in the injection of a diluted phormaline solution, in the concentration of 0.5 to $5.0 \%$, in the dorsal region of the hind paw of rats, producing a biphasic nociceptive response. In its first phase, includes the first 5 minutes after the injection, and, in a second phase starts on the $20^{\text {th }}$ minute and extends for 40 to 60 minutes. The first phase is interpreted as the result of direct activation of peripheral nociceptive receptors, while the second phase is interpreted as the result of acute inflammatory response and central sensitization. The period between both phases is identified as a phase of inactivity, being attributed to a central antinociceptive mechanism ${ }^{7,9}$. A review on the phormaline test concluded that it has considerable advantages over other tests, being considered the standard model to study nociception in animals ${ }^{10}$. Comparing to other pain-inducing agents, phormaline produces more consistent spontaneous response, offering the benefit of a biphasic response with a great potential for the study of acute pain ${ }^{11}$.

The number of paw elevations was used to quantify the painful behavior induced by phormaline, since it has an important correlation with the classical phormaline test and with the cardiovascular changes in response to the pain caused by the injection of this substance in the paw. It has a reliable correlation with pain behavior in non-physically restricted conscious animals ${ }^{11-17}$. The literature has few data on the role of serotonergic or noradrenergic receptors in the CNS in mediating response to phormaline-induced pain.

Several doses of subarachnoid phentolamine and methysergide are used in rats, and in the present study we used doses already described and that are commonly used by other authors $4,18,19$.

Pentobarbital, diazepam, and alcohol inhibit the reduction in nociception seen in the intermediate phase of the phormaline test, which is assumed to be secondary to the involvement of $\mathrm{GABA}_{A}$ receptors ${ }^{9}$. On the other hand, an electrophysiological study in rats anesthetized with sodium thiopental followed by section of the spinal cord showed biphasic behavior, suggesting that control of phormaline-induced pain does not depend of a GABAergic mechanism ${ }^{20}$.

In the present study, we observed an increase in the number of paw elevations during the intermediate phase in GF, GM, and GFM groups, which shows the involvement of serotonergic and noradrenergic pathways in the inhibitory descending pain system. According to the results observed in this study, involvement of serotonin $(5-\mathrm{HT})$ in descending pain modulation was also demonstrated through inhibition of its synthesis by $p$-chlorophenylalanine, electrolytic damage of the nucleus raphe magnus, and the use of 5,6-dihydroxytriptamine, a serotonergic neurotoxin ${ }^{21}$. Another study indicated a reduction in nociceptive response to phormaline in all phases with the use of the serotonergic agonist, $\alpha$-methyl-5-HT 22.

We expected to find an addictive or potentiating effect in the number of paw elevations with the association of serotonergic and noradrenergic antagonists. A greater number of paw elevation was observed in GMF group; however, it did not have statistical significance when compared to GF and GM groups, and the former had the lower number of paw elevations, which also did not show statistical significance. This data suggests that the dose of phentolamine was high enough to inhibit almost completely the adrenergic descending system and that the dose of methysergide was not elevated enough to inhibit completely the serotonergic descending pathway; however, the association of both drugs seems to have been enough to inhibit the descending serotonergic and adrenergic inhibitory activities. The use of logarithmically lower doses could show this effect of the serotonergic and noradrenergic antagonists, phentolamine and methysergide, as well as its association, on the inhibition of serotonergic and noradrenergic descending inhibitory activities, since the data of the present study allows one to infer that, despite having use doses evaluated by other authors $4,18,19$, they could have been higher enough to elucidate controversial aspects of the serotonergic and noradrenergic inhibitory descending activities. 
On the other hand, a study with several pain models observed an antinociceptive effect of methysergide in the second phase of the phormaline test, suggesting that is due to the reduced release of glutamate and substance $\mathrm{P}$ by primary afferent neurons in the spinal cord caused by the blockade of 5-HT2 receptors prior to peripheral inflammation ${ }^{23}$. On the other hand, the inhibitory role of noradrenergic pathways was demonstrated in the model of tail withdrawal, in which phentolamine and yohimbine led to an increase in the electrical stimulation of the lateral reticular nucleus to activate the inhibitory descending system ${ }^{24}$.

Another study observed a reduction in the nociceptive response of the phormaline test, with yohimbine, and a relatively selective adrenergic $\alpha 2$ antagonist and serotonergic $5-\mathrm{HT} 1 \mathrm{~A}$ receptors, allowing the inference of the direct involvement of $\alpha 1$ receptors in the modulation of nociception in phase II of the phormaline test, since this was observed with phentolamine, an unspecific antagonist of $\alpha 1$ and $\alpha 2$ reecptors ${ }^{25}$.
In this study, during phase II of the modified phormaline test, a significant difference was observed in nociceptive response when the phentolamine and phentolamine associated with methysergide groups were compared to the methysergide group, which might be explained by the antagonistic effect of phentolamine in $\alpha 2$ receptors, promoting a $\alpha 1$ effect.

The results observed indicate an increase in pain response with the use of subarachnoid noradrenergic and serotonergic antagonists in rats, which suggests the presence of a noradrenergic and serotonergic effects in the descending inhibitory system of acute pain, indicating the importance of investigating agonists that might present analgesic effects in this and other pain models, therefore allowing its use in the control of acute pain. Despite this, some ongoing studies performed by another investigator of our institution, adopting other experimental pain models should confirm our findings. 